ENCYCLOPÉDIE Encyclopédie berbère

BERBERE

9 | 1991

9 | Baal - Ben Yasla

\title{
Basilique chrétienne africaine
}

\section{N. Duval}

\section{OpenEdition}

Journals

Édition électronique

URL : http://journals.openedition.org/encyclopedieberbere/1299

DOI : 10.4000/encyclopedieberbere.1299

ISSN : 2262-7197

\section{Éditeur}

Peeters Publishers

\section{Édition imprimée}

Date de publication : 1 avril 1991

Pagination : 1371-1377

ISBN : 2-85744-509-1

ISSN : 1015-7344

\section{Référence électronique}

N. Duval, « Basilique chrétienne africaine », Encyclopédie berbère [En ligne], 9 | 1991, document B42, mis en ligne le 01 décembre 2012, consulté le 24 septembre 2020. URL : http://journals.openedition.org/ encyclopedieberbere/1299; DOI : https://doi.org/10.4000/encyclopedieberbere.1299

Ce document a été généré automatiquement le 24 septembre 2020

(c) Tous droits réservés 


\title{
Basilique chrétienne africaine
}

\author{
N. Duval
}

1 Le but de cet article est de déterminer s'il existe un type de basilique chrétienne propre à l'Afrique et qui a pu être influencé par des traditions locales, voire « indigènes ».

2 L'essentiel de cette typologie est établi depuis le début du $\mathrm{xx}^{\mathrm{e}}$ siècle avec des synthèses de St. Gsell pour l'Algérie, en 1901, un recueil de plans d'églises tunisiennes réunis par P. Gauckler, publié en 1913 et commenté par P. Monceaux, un article de Dom H. Leclercq dans le Dictionnaire d'archéologie chrétienne et de liturgie. De nouvelles mises au point ont été faites en 1938 par le P. Lapeyre dans un rapport sur la Tunisie, en 1953 par J.-B. Ward Perkins et R.-G. Goodchild à propos de la Tripolitaine, en 1970 par J. Lassus, puis par P.-A. Février, N. Duval et J. Christern.

3 Le type de la "basilique africaine » peut être considéré comme illustré par une curieuse image sur une tombe en mosaïque dans une église de Tabarka (fig. 1) qui symbolise l' Ecclesia Mater, l'Église Mère qui accueillera la défunte dans l'Au-Delà. 
Dessin de la mosaïque de l'Ecclesia Mater à Tabarka et reconstitution de labasilique représentée (d'après Ward Parkins). La restitution d'une architrave à la place d'arcades est due à une mauvaise interprétation de l'image.
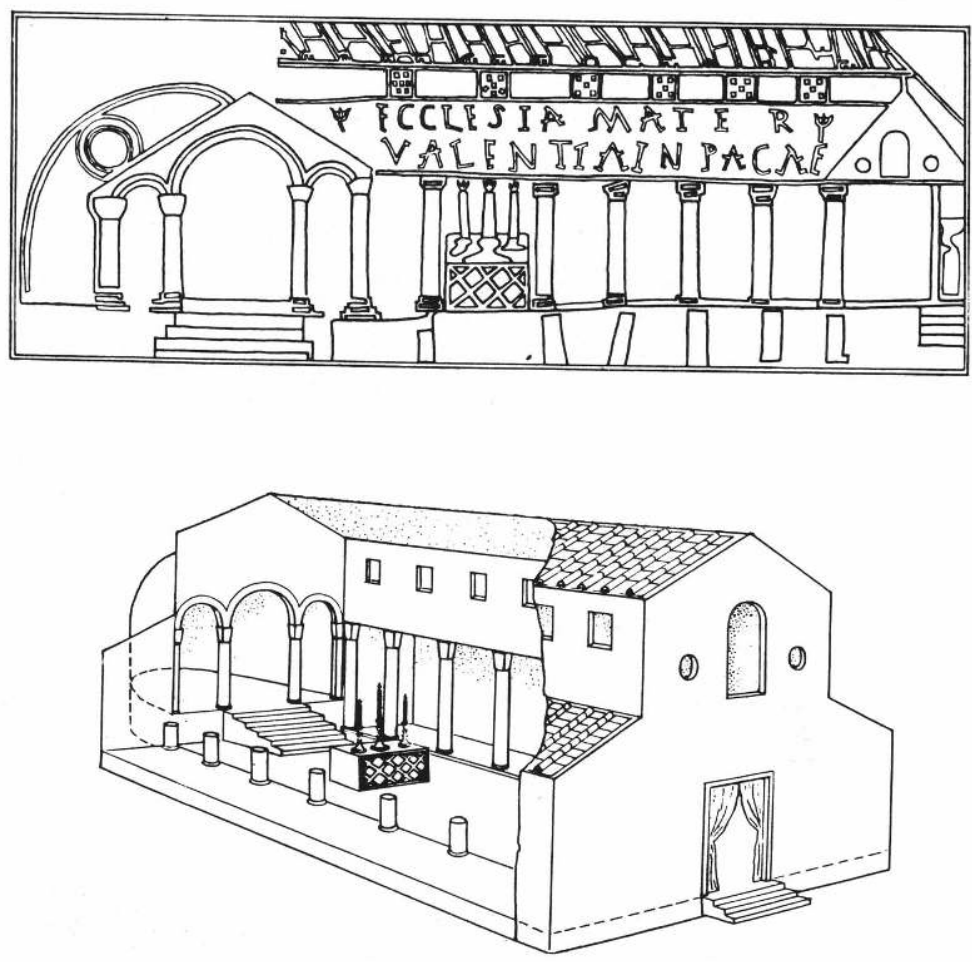

4 Il s'agit de la représentation d'une basilique décomposée où l'on a juxtaposé côte à côte, en largeur, la façade, les nefs et l'abside, et en hauteur - séparées par une brève épitaphe - une vue transversale sur le quadratum populi (c'est-à-dire les nefs de l'église dont on a supprimé le mur latéral et coupé la première colonnade) et la toiture de la nef centrale: ce type d'image "synthétique", rompant délibérément avec la perspective, est destiné à montrer le plus de faces possible du même bâtiment, à figurer l'intérieur en même temps que l'extérieur suivant l'intérêt de chaque élément; ce procédé est employé fréquemment depuis l'Antiquité tardive jusqu'au Moyen Age.

A travers cette figuration célèbre et les plans rassemblés dans les recueils précités (300 monuments environ au total), on peut affirmer que, sans rompre avec le type traditionnel de la «basilique latine » illustré par les grandes basiliques de Rome (SaintPierre, Saint-Paul, Saint-Jean-de-Latran, Sainte-Marie Majeure), la basilique africaine présente certaines particularités (fig. 2 et 3 ). Son orientation paraît très variable mais un noyau important d'églises possédant leur abside à l'ouest existe dans tout l'est de l'Afrique du Nord. Cette "occidentation» était aussi une des caractéristiques des grandes églises romaines alors que l'orientation domine dans l'est de l'Empire. 
Vue cavalière du chœur et de l'abside d'une basilique africaine (d'après van der Meer) : remarquer l'autel dans la nef et l'abside surélevée avec le synthronos et la cathèdre épiscopale.

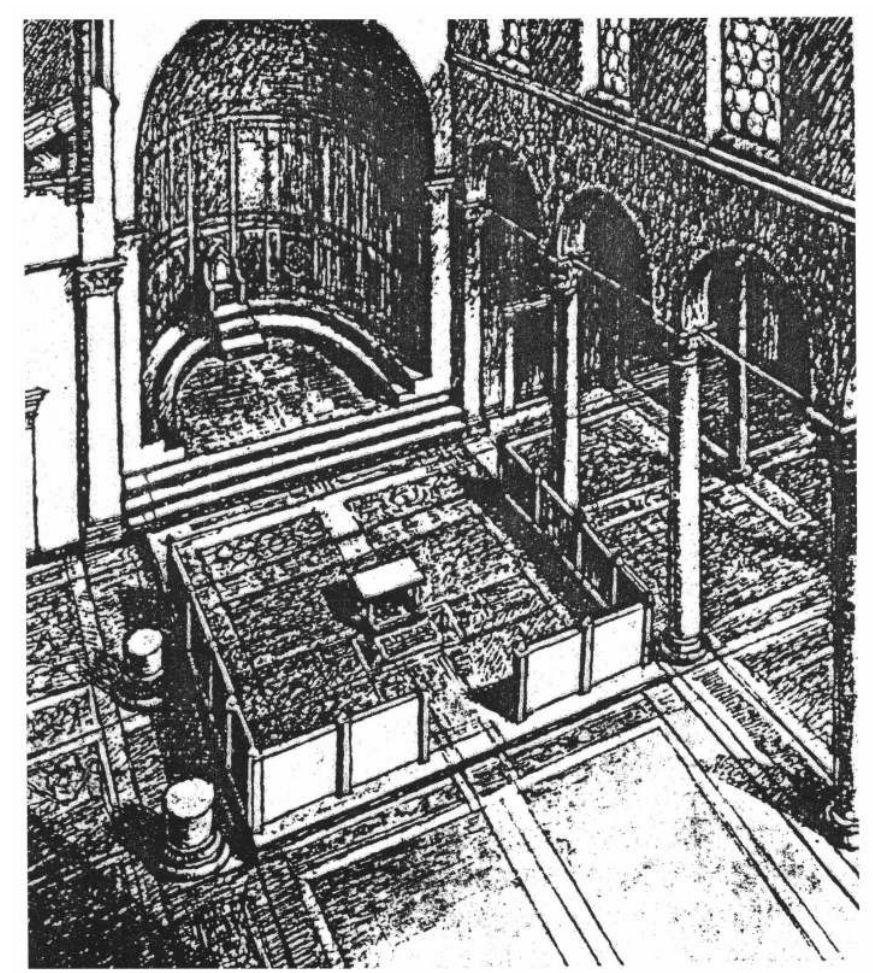

6 Dans la plupart des cas l'église africaine est dépourvue d'atrium (mais les grandes églises de Carthage en comportent un): on entre directement dans l'église par l'intermédiaire d'un portique ou d'un vestibule plutôt qu'un narthex (terminologie, forme architecturale et usage liturgique propres à l'orient).

7 Le type le plus courant possède trois nefs séparées par des colonnades à arcades (contrairement à ce que pourrait faire croire la mosaïque de Tabarka qui semble montrer des architraves, qui sont de simples lignes de séparation) supportant une "claire-voie», avec couverture en charpente: aucune particularité ici sinon dans l'utilisation fréquente de supports doubles, deux colonnes accolées ou une colonne associée à un pilier dans 1'« École de Tébessa » 
Plan de la basilique V de Thélepte (Tunisie). Remarquer le plan du « chœur » avec l'emplacement de l'autel et le chevet tripartite.

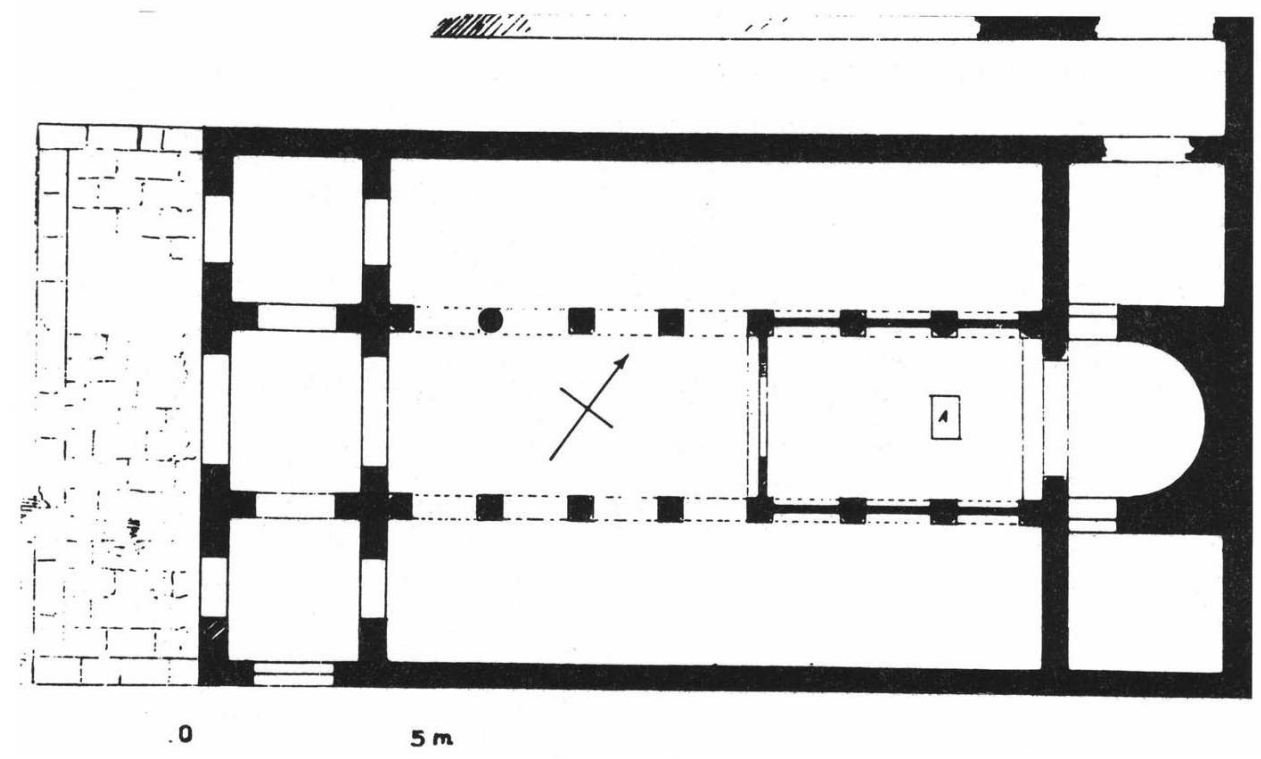

8 C'est dans l'organisation interne que se manifeste une spécificité « africaine » : l'autel, qui dans d'autres régions est situé dans l'abside ou à la corde de l'abside, est placé ici au milieu du " peuple » mais protégé par une enceinte particulière, très avancée dans la nef à l'époque ancienne, se rapprochant progressivement de l'abside au $\mathrm{VI}^{\mathrm{e}}$ siècle. D'autre part, il est associé régulièrement (ce n'est pas le cas par exemple en Syrie) à des reliques qui sont placées généralement dans la base ou sous la base du meuble liturgique, dans un loculus. Une organisation complexe des cheminements et des barrières découle de cet emplacement, surtout quand un second « centre de culte » (un autre autel ou une contre-abside abritant souvent aussi des reliques ou un corps saint) se situe à l'entrée de la nef centrale ou contre la façade comme c'est le cas dans une trentaine de monuments alors que cette disposition est très rare ailleurs (quelques cas en Espagne). Il peut se faire qu'un couloir axial relie les différentes enceintes réservées aux mouvements du clergé ou même que toute la nef centrale soit interdite à l'accès des fidèles par des «chancels ». En revanche, on ne trouve pas dans le Maghreb (mais on le connaît en Tripolitaine) un autre aménagement qui permet la prise de parole ou les lectures au milieu du "peuple»: l'ambon, qui est de règle en Orient. Son absence est liée sans doute à l'organisation liturgique qui privilégie l'abside pour ce rôle (voir infra).

9 A l'extrémité de la nef centrale, sans l'intermédiaire d'un transept, l'abside couverte d'une demi-coupole est, en effet, généralement surélevée en Afrique, parfois de plus d'un mètre (sans que la présence d'une crypte explique dans la plupart des cas cette dénivellation); elle est par conséquent accessible par un escalier, soit des marches occupant toute la largeur, soit un perron axial, soit deux volées de marches latérales. C'est là que siègent les prêtres: une banquette (ou plusieurs gradins superposés) longeant le mur en demi-cercle devait exister à peu près partout. Pendant longtemps on n'en a pas reconnu de vestiges et on a conclu à des bancs en bois. Les fouilles récentes ont révélé de plus en plus de restes de ces installations mais la maçonnerie, légère et fragile, avait pu échapper aux dégagements un peu brutaux des premiers fouilleurs. Au milieu de la banquette, une cathèdre surélevée accueille l'évêque, au 
moins dans les villes qui sont le siège d'un évêché (mais c'est très souvent le cas puisque chaque cité africaine disposant d'une autonomie municipale a pratiquement un évêque comme chef de la communauté). C'est de ce siège, devenu le symbole du pouvoir épiscopal (les mots sedis et cathedra servent à le désigner), que l'évêque parle à son peuple, à sa plebs comme nous l'apprennent par exemple les nombreuses allusions des sermons de St. Augustin. La surélévation de l'abside et l'inutilité d'un ambon sont liées sans doute à cette pratique.

Plan du dernier état de la basilique II de Sbeitla (Tunisie). Remarquer la contre-abside avec un autel, la nef centrale presque entièrement isolée avec l'autel au centre et le baptistère derrière le presbyterium.

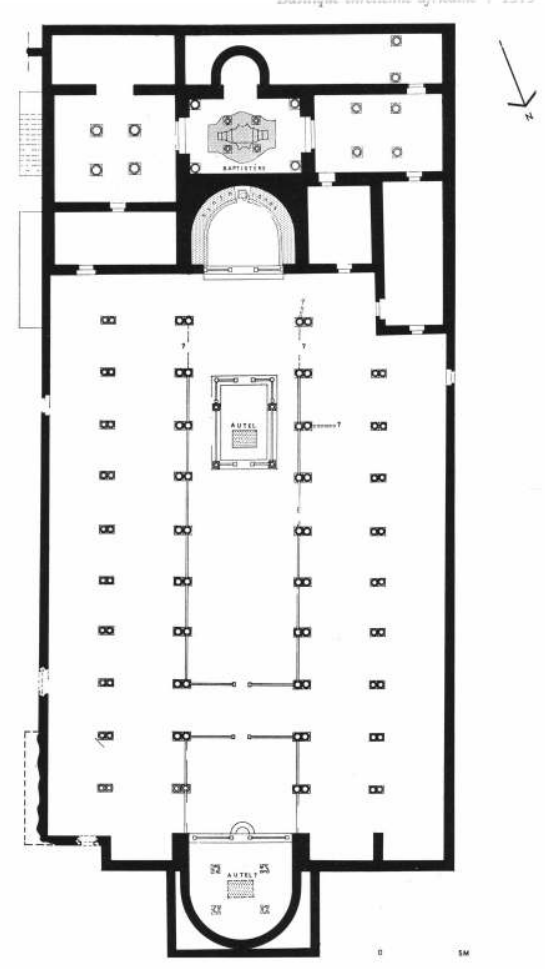

10 Le chevet des églises africaines a toujours été considéré comme une des caractéristiques essentielles de l'architecture cultuelle de ce pays et souvent - mais à tort - comme un signe d'influence syrienne : l'abside est prise dans beaucoup de cas dans un massif rectangulaire et encadrée par deux pièces complémentaires si bien que le mur du chevet est rectiligne (mais naturellement la couverture distingue la demicoupole de l'abside, extradossée ou protégée par une toiture, et les appentis qui, à un niveau inférieur, servent normalement à abriter les pièces latérales). La même disposition se retrouve effectivement souvent en Syrie, mais l'Afrique ne paraît pas avoir conçu la même utilisation d'un plan comparable. Les pièces latérales, qui communiquent avec l'abside et/ou avec les nefs, que les spécialistes ont pris l'habitude - utilisant un vocabulaire oriental - d'appeler prothesis et diaconicon et que nous préférons désigner d'un terme plus neutre de "sacristies ", n'ont pas ici d'affectation déterminée. Si certaines ont servi probablement de salles de préparation pour le clergé et les actes liturgiques, ou de réserves pour le matériel, dans d'autres cas on y a installé un baptistère ou inhumé des défunts. Rappelons aussi que les statistiques montrent que 
ce plan-type « africain » connaît de nombreuses variantes et que la moitié environ des monuments ne s'y conforment pas.

11 L'église est souvent associée à un baptistère ou à un complexe de salles servant à la cérémonie du baptême, même quand il ne s'agit pas d'une cathédrale puisque, à partir $\mathrm{du} \mathrm{V}^{\mathrm{e}}$ siècle au moins, le baptême ne paraît plus réservé exclusivement à l'évêque. L'emplacement et la forme du baptistère sont extrêmement variés encore qu'une petite série (en Byzacène et en Tripolitaine surtout) semble préférer un agencement prolongeant le chevet de l'église par un «bloc» de même largeur où le baptistère proprement dit, situé derrière l'abside, est encadré par deux salles annexes. La forme de la vasque a suivi une évolution parallèle à celle que nous trouvons dans d'autres régions : formes simples - ronde ou carrée - remplacées progressivement par un plan polygonal puis cruciforme ou polylobé (ce dernier est presque exclusivement réservé à l'Afrique et à la période byzantine). Le bassin n'est pas très profond, il est souvent dépourvu de dispositifs d'adduction et d'évacuation: le rite de l'immersion est peutêtre devenu symbolique ou a été complété par l'aspersion.

12 Les traits particuliers de la basilique africaine qui ont ainsi été définis s'expliquent-ils par des traditions locales? Non pas, si l'on parle d'une tradition architecturale venue de l'époque punique, numide ou romaine. Les structures du bâtiment sont classiques dans le contexte de l'architecture chrétienne. Elles sont naturellement apparentées à celles de la basilique civile, même pour le type à deux absides opposées, et il est arrivé à plusieurs reprises (notamment à Tipasa et Lepcis Magna) que des églises aient été installées dans des «basiliques» antérieures qui, d'ailleurs, n'avaient rien de spécifiquement africain. Il existait par contre un type de temple "africain", généralement à cella de petites dimensions (carrée ou rectangulaire), au fond d'une cour, mais le sanctuaire était le plus souvent trop petit pour les nécessités du culte chrétien et, quand celui-ci s'y est installé, on a aménagé à plusieurs reprises le baptistère dans la cella en construisant l'église dans la cour ou au voisinage.

13 Mais l'Église d'Afrique, d'origine très ancienne et marquée au ${ }^{\mathrm{e}}{ }^{\mathrm{e}}$ siècle par sa volonté de préserver ses traditions en face du Siège Apostolique, possède indiscutablement un rituel et une liturgie qui lui sont propres et qui ont gardé leur spécificité pendant plusieurs siècles. Bien que nous les connaissions mal puisqu'il nous manque les textes techniques qui existent par exemple pour l'Italie, la Gaule, l'Espagne, ces rites peuvent se reconnaître au moins dans leurs conséquences matérielles qui ont influencé l'emplacement de l'autel, l'aménagement de l'abside, l'adjonction éventuelle d'une contre-abside, sans doute l'organisation du chevet de l'église, peut-être la forme de la vasque baptismale.

14 L'importance en Afrique du culte des martyrs, bien connue par les textes des Pères, explique la présence régulière de reliques sous l'autel et sans doute certains aménagements comme des monuments commémoratifs - memoriae ou cénotaphes ou les « contre-absides », particulièrement fréquentes en Afrique.

15 Elle peut rendre compte aussi d'une autre spécificité des églises africaines qui accueillent régulièrement, même en ville, dans leur sol des sépultures que la loi romaine proscrivait autrefois dans l'enceinte des cités et que d'autres régions ont toujours répugné à accepter dans l'édifice cultuel (même en dehors de la ville). Ces inhumations qui se pressent surtout ad sanctos, à proximité des reliques, ont fait naître une mode, qui n'est pas uniquement mais plus spécialement africaine, de «mosaïques 
funéraires " pour concilier la nécessité de signaler la sépulture et celle de conserver un pavement adapté aux préférences locales.

La forte originalité de l'Afrique chrétienne - à travers la discipline ecclésiastique, la place faite aux martyrs et aux défunts, les rites et la liturgie - a donc fait naître une typologie particulière, plus dans les aménagements que dans l'architecture proprement dite. Malgré une grande diversité locale, surtout dans les techniques de construction et les décors influencés souvent par des ateliers régionaux, il ne semble pas qu'on puisse affirmer, comme certains l'ont fait (W.H.C. Frend) que cette typologie est mieux représentée dans les zones "de tradition berbère ", dans les campagnes de l'Afrique profonde (par exemple en Numidie centrale et méridionale) que dans les villes les plus romanisées. Le schisme, africain par excellence, du Donatisme n'a pas fait naître une architecture religieuse qui lui soit propre, ou du moins n'est-on pas capable de la reconnaître dans l'état actuel des recherches.

A priori, par conséquent, la «basilique africaine » doit plus à une tradition liturgique dont il faut faire remonter la naissance à la christianisation des II $^{e}$ et III $^{e}$ siècles, qu'à des influences ethniques ou à un héritage architectural des civilisations du Maghreb.

\section{BIBLIOGRAPHIE}

BERTHIER A., LOGEART F. et MARTIN M., Les vestiges du christianisme en Numidie centrale, Alger, s.d. (1942). CHRISTERN J., Das frühchristliche Pilgerheiligtum von Tebessa, Wiesbaden, 1976.

DUVAL N., « Église et temple en Afrique », Bull, archéol. du Comité des Trav. Hist., n.s., 1971, 7, p. 265-296 ; « L'architecture chrétienne en Byzacène (Recherches d'architecture chrétienne nordafricaine, II) », Mélanges de l'École Française de Rome, Antiquités, 1972, 84, p. 1127-1171.

DUVAL N. et FÉVRIER P.-A., « Le décor des monuments chrétiens d'Afrique (Algérie-Tunisie) », Actas del VIII Congr. int. de Arqueologia Cristiana, Barcelona, 1969, Rome, 1972, p. 5-55.

DUVAL N., Les églises africaines à deux absides, II, Paris, 1973.

DUVAL N., « L'évêque et la cathédrale en Afrique du Nord », Actes du XI Congr. d'Archéol. chrétien., Lyon, 1986, Rome, 1989, 1.1, p. 345-403.

GUI I., sous la direction de N. Duval, avec la participation de J.P. Caillet, Typologie des églises d'Afrique du Nord, Paris, 1991.

FÉVRIER P.-A., « Les sources épigraphiques et archéologiques de l'histoire religieuse des provinces orientales de l'Afrique antique », Corsi Ravenna, 1972, XIX, p. 131-158.

FREND W.-H.-C, The Donatist Church, Oxford, 1952.

GAUCKLER P., Basiliques chrétiennes de Tunisie, Paris, 1913 (recueil de plans avec préface de P. Monceaux).

GSELL St., Les monuments antiques de l'Algérie, t. II, Paris, 1901 (en particulier la synthèse des p. 113-156). 
LAPEYRE G.-G., « La basilique chrétienne en Tunisie », Atti del IV Congr. int. di Archeologia cristiana, 1938, I, Rome, 1940, p. 169-244.

LASSUS J., « La basilique africaine ; les baptistères africains ", Corsi di cultura sull'Arte ravennate e bizantina, 1970, XVII, p. 217-252.

LASSUS J., " L'architecture chrétienne de l'Afrique du Nord », Actas del VIII Congr. de Arqueo-logia Cristiana, Barcelona, 1969, Rome, 1972, p. 107-125.

LECLERCQ H., Dictionnaire d'archéologie chrétienne et de liturgie, s.v. « Afrique » (Archéologie de 1'), Paris, 1924, col. 658-747.

MONCEAUX P., Histoire littéraire de l'Afrique chrétienne, 7 vol. parus, Paris, 1901-1923.

ROMANELLI P., « Topografia e archeologia dell'Africa romana », Enciclopedia classica, t. X, 7, Turin, 1970, p. 349-407.

WARD PERKINS et GOODCHILD R.-G., " The Christian Antiquities of Tripolitania », Archeologia, XCV, 1953 (en particulier p. 56-78).

INDEX

Mots-clés : Antiquité, Architecture, Christianisme, Religion 